1 Rare Variants in the $O T O G$ Gene Are a Frequent Cause of Familial

\title{
Meniere's Disease
}

3 Pablo Roman-Naranjo ${ }^{1}$, Alvaro Gallego-Martinez ${ }^{1}$, Andrés Soto-Varela ${ }^{2}$, Ismael Aran ${ }^{3}$,

4 Maria del Carmen Moleon ${ }^{4}$, Juan Manuel Espinosa-Sanchez ${ }^{4}$, Juan Carlos Amor-

5 Dorado $^{5}$, Angel Batuecas-Caletrio ${ }^{6}$, Paz Perez-Vazquez $^{7}$ and Jose A. Lopez-Escamez ${ }^{1,4}$

$6{ }^{1}$ Otology \& Neurotology Group CTS 495, Department of Genomic Medicine, Centro

7 Pfizer-Universidad de Granada-Junta de Andalucía de Genómica e Investigación

8 Oncológica, Granada, Spain

$9 \quad{ }^{2}$ Division of Otoneurology, Department of Otorhinolaryngology, Complexo

10 Hospitalario Universitario, Santiago de Compostela, Spain.

$11{ }^{3}$ Department of Otolaryngology, Complexo Hospitalario de Pontevedra, Pontevedra,

12 Spain.

$13{ }^{4}$ Department of Otolaryngology, Instituto de Investigación Biosanitaria,

14 ibs.GRANADA, Hospital Universitario Virgen de las Nieves, Granada, Spain.

$15{ }^{5}$ Department of Otolaryngology, Hospital Can Misses, Ibiza, Spain.

$16{ }^{6}$ Department of Otolaryngology, Hospital Universitario Salamanca, Instituto de

17 Investigación Biomédica de Salamanca (IBSAL), Salamanca, Spain.

$18{ }^{7}$ Department of Otorhinolaryngology, Hospital Universitario de Cabueñes, Gijón, Spain.

20 All correspondence should be addressed to:

21 José Antonio López Escámez, Otology \& Neurotology Group CTS495, GENYO, -

22 Centre for Genomics and Oncological Research- Pfizer/Universidad de

23 Granada/Andalusian Regional Government, Avda de la Ilustración 114, Granada 18016

24 Spain. E-mail: antonio.lopezescamez@genyo.es Phone. +34 958715 500-160

\section{Conflicts of Interest and Source of Funding:}

27 Jose Antonio Lopez Escamez (JALE) is partially funded by INT18/00031 from ISCIII.

28 This study was funded by the Luxembourg National Research Fund 
bioRxiv preprint doi: https://doi.org/10.1101/771527; this version posted September 21, 2019. The copyright holder for this preprint (which was not certified by peer review) is the author/funder, who has granted bioRxiv a license to display the preprint in perpetuity. It is made available under aCC-BY-NC-ND 4.0 International license.

29 INTER/Mobility/17/11772209 Grant and EF-0247-2017 from Andalusian Health

30 Government to JALE. Authors declare no conflict of interest. 


\section{Abstract}

32 Objectives: Meniere's disease (MD) is a rare inner ear disorder characterized by

33 sensorineural hearing loss, episodic vertigo and tinnitus. Familial MD has been reported

34 in 6-9\% of sporadic cases, and few genes including FAM136A, DTNA, PRKCB,

35 SEMA3D and DPT have been involved in single families, suggesting genetic

36 heterogeneity. In this study, the authors recruited 46 families with MD to search for

37 relevant candidate genes for hearing loss in familial MD.

38 Design: Exome sequencing data from MD patients were analyzed to search for rare

39 variants in hearing loss genes in a case-control study. A total of 109 patients with MD

40 (73 familial cases and 36 early-onset sporadic patients) diagnosed according to the

41 diagnostic criteria defined by the Barany Society were recruited in 11 hospitals. The

42 allelic frequencies of rare variants in hearing loss genes were calculated in individuals

43 with familial MD. A single rare variant analysis (SRVA) and a gene burden analysis

44 (GBA) were conducted in the dataset selecting one patient from each family. Allelic

45 frequencies from European and Spanish reference datasets were used as controls.

46 Results: A total of 5136 single nucleotide variants in hearing loss genes were

47 considered for SRVA in familial MD cases, but only one heterozygous variant in the

48 OTOG gene (rs552304627) was found in two unrelated families. The GBA found an

49 enrichment of rare missense variants in the $O T O G$ gene in familial MD. So, 15/46

50 families (33\%) showed at least one rare missense variant in the OTOG gene, suggesting

51 a key role in familial MD.

52 Conclusions: The authors found an enrichment of multiplex rare missense variants in

53 the $O T O G$ gene in familial MD. This finding supports $O T O G$ as a relevant gene in

54 familial MD and set the groundwork for genetic testing in MD. 


\section{INTRODUCTION}

56 Meniere's disease [MD (OMIM 18600)] is a rare inner ear disorder with three major

57 symptoms: sensorineural hearing loss (SNHL), episodic vertigo and tinnitus (Lopez-

58 Escamez et al. 2015; Espinosa-Sanchez \& Lopez-Escamez 2016). Hearing loss always

59 involves low and medium frequencies in one or both ears (unilateral or bilateral MD) at

60 the onset of the disease. However, MD also affects high frequencies in early or

61 advanced stages of the disease (Belinchon et al. 2011). Epidemiological studies indicate

62 that MD is most common in European population, suggesting a genetic predisposition

63 (Ohmen et al. 2013). Although the majority of MD patients are considered sporadic

64 (Frejo et al. 2016; Frejo et al. 2017), familial clustering has been reported in 8-9\% of

65 sporadic cases in the European descendent (Requena et al. 2014), and in 6\% of Korean

66 population (Lee et al. 2015), which also supports a genetic contribution to the disease

67 (Roman-Naranjo et al. 2017). MD shows a wide range of phenotypic variations among

68 patients, even within the same families (Lee et al. 2015b), and it is commonly

69 associated with migraine and systemic autoimmune disorders (Tyrrell et al. 2014; Cha

70 et al. 2008). Familial MD (FMD) shows an autosomal dominant (AD) pattern of

71 inheritance with incomplete penetrance and anticipation, showing an earlier onset

72 compared to sporadic cases (Morrison et al. 2009; Birgerson et al. 1987; Klar et al.

73 2006). Different whole exome sequencing (WES) based studies have identified several

74 genes related with FMD. Single nucleotide variants (SNV) in DTNA, FAM136A,

$75 P R K C B, D P T$ and $S E M A 3 D$ were identified in 4 different families AD inheritance with

76 incomplete penetrance (Requena et al. 2015; Martín-Sierra et al. 2016; Martín-Sierra et

77 al. 2017). However, these findings have not been replicated neither in other MD

78 families nor sporadic MD (SMD) cases. 
79 WES continues to be an efficient tool to determine disease-causing variants (Williams et

80 al. 2016; Adams \& Eng 2018; Suwinski et al. 2019), although the monogenic

81 hypothesis in FMD should be reconsidered to achieve results beyond private rare

82 variants for singular families. Thus, the "one variant-one disease" hypothesis, described

83 for classic Mendelian inheritance cannot explain the incomplete penetrance or variable

84 expressivity observed in MD (Martín-Sierra et al. 2017) and more complex inheritance

85 models are needed (Cooper et al. 2013; Kousi \& Katsanis 2015). Oligogenic and

86 multiallelic models have been already applied in different diseases, such as Parkinson

87 (Lubbe et al. 2016) and Huntington's disease (Lee et al. 2015a), explaining changes in

88 disease progression and phenotypic variability. Furthermore, a digenic inheritance of

89 deafness was reported by variants in $C D H 23$ and PCDH15 (Zheng et al. 2005), and

90 recently, an enrichment of rare missense variants in certain SNHL genes, such as GJB2,

$91 S L C 26 A 4$ or $U S H 1 G$, was found in a large cohort of SMD cases (Gallego-Martinez et

92 al. 2019), supporting the hypothesis of multiallelic inheritance in MD.

93 More than 150 genes have been associated to deafness (Azaiez et al. 2018), and 116 of

94 them are related with non-syndromic SNHL (Van Camp G. 2018).

95 In this study, we have investigated the genetic background of FMD, focusing on SNHL

96 genes by analyzing 46 families with MD by WES. We have found an enrichment of rare

97 missense variants in the $O T O G$ gene compared with non-Finnish European (NFE) and

98 Spanish populations. The $O T O G$ gene, which encodes otogelin, has been previously

99 associated with deafness and imbalance and causes autosomal recessive deafness $18 \mathrm{~B}$

100 (Simmler et al. 2000a; Schraders et al. 2012). A total of 15 families out of 46 showed, at

101 least, one rare missense variant in this gene, suggesting a key role of otogelin in MD. 


\section{MATERIALS AND METHODS}

\section{Patient assessment and selection}

\section{4}

A total of $73 \mathrm{MD}$ patients from 46 different families with one or more affected firstdegree relatives, and 36 sporadic MD cases with an age of onset younger than 35 were recruited. Patients were diagnosed following the diagnostic criteria described by the International Classification Committee for Vestibular Disorders of the Barany Society (Lopez-Escamez et al. 2015). A complete hearing and vestibular assessment was carried out in all cases, including a brain magnetic resonance imaging to exclude other causes of neurological symptoms. Serial pure tone audiograms were retrieved from clinical records to assess hearing loss since the initial diagnosis. A summary of the clinical information of these patients is presented in the Supplemental Digital Content 1 (see Table 1 to Table 3, Supplemental Digital Content 1).

This study protocol was approved by the Institutional Review Board for Clinical Research (MS/2014/02), and a written informed consent to donate biological samples was obtained from all subjects.

\section{DNA extraction and whole exome sequencing}

Blood and saliva samples were taken from patients with MD to perform WES. DNA samples were extracted with prepIT-L2P (DNA Genotek, Ottawa, Canada) and QIAamp DNA Blood Mini Kit (Qiagen, Venlo, The Netherlands) using manufacturer's protocols and quality controls previously described (Szczepek et al. 2019). DNA libraries were prepared by using the SureSelect Human All Exon V6 kit (Agilent Technologies, Santa Clara, CA, USA) and were paired-end sequenced on the Illumina HiSeq 4000 platform at 100X coverage. Raw reads were stored in two FASTQ files for each individual. 


\section{Bioinformatic analysis}

\section{Dataset generation and processing}

127 Analysis-ready BAM files and VCF files were generated from raw unmapped reads

128 using the GATK Best Practices pipeline. Reads were aligned to the GRCh37/hg19

129 human reference genome using the BWA-MEM algorithm. For obtaining the final

130 dataset, SNV and small structural variants were filtered according to its Variant Quality

131 Score Recalibration (VQSR) and depth of coverage (DP) values. Thus, variants were

132 excluded if their VQSR value were under the VSQR threshold or their average DP $<10$.

133 Variants were functionally annotated using ANNOVAR version 2018Apr16. RefSeq

134 was used for gene-based annotation and the Exome Aggregation Consortium (ExAC)

135 database, the Combined Annotation Dependent Depletion (CADD) scores and the

$136 \mathrm{dbNSFP}$ database (v3.0) were used for filter-based annotation.

\section{Sensorineural hearing loss gene set}

138 The SNHL gene set was generated by using three different databases: the Hereditary

139 Hearing Loss Homepage (Van Camp G. 2018), the Deafness Variation Database

140 (Azaiez et al. 2018) and Harmonizome (Rouillard et al. 2016), containing a total of 116

141 genes related with SNHL (see Table 4, Supplemental Digital Content 1).

\section{Data analysis and prioritization strategy}

143 Two pipelines and filtering/prioritization strategies were conducted to search for rare

144 variants as we have previously described (Gallego-Martinez et al. 2019). The first was a

145 single rare variant analysis (SRVA) for studying individual families; the second

146 approach was a gene burden analysis (GBA) to obtain a gene-level mutational profile

147 (Figure 1). For these analyses only one patient from each family was selected. 
148 Whenever possible, the patient selected was in the last generation. Sporadic cases with

149 an early onset were also investigated to search for singleton variants in candidate genes

150 in both analyses.

151 All variants were assessed according to the standards and guidelines described by the

152 American College of Medical Genetics and Genomics (ACMG) and the Association for

153 Molecular Pathology (AMP) (Richards et al. 2015). Variants not described in the NFE

154 population from ExAC and the Spanish population from CSVS were discarded to

155 minimize false calls and population-specific variants (Shearer et al. 2014). Selected

156 variants were checked in patients BAM files with IGV and/or sequenced by Sanger

157 sequencing to minimize false calls.

\section{Statistics and databases}

159 Two independent datasets were used as reference to compare the observed MAF in

160 FMD and to calculate odds ratios (OR): NFE population from ExAC and the

161 Collaborative Spanish Variant Server (CSVS) database (Lek et al. 2016; Dopazo et al.

162 2016).

163 For each selected variant in the SRVA, OR with 95\% confidence interval (CI) were

164 calculated using the MAF values from the CSVS database $(\mathrm{N}=1,579)$ and the NFE 165 population $(\mathrm{N}=33,365)$ from ExAC.

166 For GBA, we counted the total exonic alternate alleles per gene in our cohort against the 167 two reference datasets. After calculating OR with 95\% CI, we obtained one-sided p168 values that were corrected for multiple testing by the total number of variants found in 169 each gene following the Bonferroni approach.

170 Standard audiometric evaluations for air and bone conduction elicited by pure tones

171 from 125 to $8000 \mathrm{~Hz}$ were retrieved from the clinical records to analyse the time course 
bioRxiv preprint doi: https://doi.org/10.1101/771527; this version posted September 21, 2019. The copyright holder for this preprint (which was not certified by peer review) is the author/funder, who has granted bioRxiv a license to display the preprint in perpetuity. It is made available under aCC-BY-NC-ND 4.0 International license.

172 of the hearing profile in FMD cases with candidate variants. Regression analysis was

173 performed to estimate the outcome of hearing loss for each frequency. 


\section{RESULTS}

\section{Main genetic findings in familial MD}

\section{Single rare variant analysis}

177 A total of 5136 variants located in SNHL genes were considered in FMD cases. After applying quality controls (QC), 4247 SNV remained. Only 75 nonsynonymous or splice site SNV fulfilled the MAF (<0.001) and CADD (>15) filtering criteria (Figure 1). From them, $40 \mathrm{SNV}$ were already described in the NFE population or Spanish population, but only one SNV was found in more than one family (see Table 5,

182 Supplemental Digital Content 1, which shows the rare variants found in the SRVA for 183 FMD cases). This heterozygous variant located in $O T O G$ gene was observed in cases from two unrelated families (F1 \& F14). The variant chr11:17574758G>A (rs552304627; p.V141M), which is in the last nucleotide of the fourth exon in the OTOG canonical transcript (ENST00000399391), is likely pathogenic according to the ACMG and AMP guidelines. This multiplex variant is located in a Von Willebrand Factor D-type domain (vWD) with a MAF=.0008 in NFE population, and multiple in silico tools supported a likely pathogenic effect of this variant (SIFT score=.001; M-

$\mathrm{CAP}=.153 ; \mathrm{CADD}=28.2 ; \mathrm{GERP}++=5.36)$.

The rest of the rare SNV were considered private familial variants because none of them were found in other FMD cases. None small structural variant (insertion or deletion) was found in any SNHL genes.

\section{Gene burden analysis}

195 Seventy-three genes with $214 \mathrm{SNV}$ with a MAF $<0.05$ were retained after QC and 196 filtering steps. Most of the genes (74\%) carried less than 3 variants, thus they were 
197 discarded for further analysis. The most significant finding was an enrichment of rare

198 missense variants in $O T O G$ gene in our FMD cases against either NFE population from

$199 \operatorname{ExAC}\left(\mathrm{OR}=4.3(2.6-7.0), p=4.1 \times 10^{-8}\right)$ or Spanish population $(\mathrm{OR}=3.6(2.1-5.9), p=$

$\left.2007.1 \times 10^{-6}\right)$. Nine different rare missense variants were found in OTOG in 14/46 non-

201 related families, existing 6 families with 2 or more shared variants (Table $1 \&$ see

202 Figure 1, Supplemental Digital Content 2). The variants rs61978648 and rs61736002

203 were shared by individuals from 4 unrelated families (F2, F3, F4 \& F5). Likewise, the

204 variants rs552304627 and rs117315845 were found in patients from other 2 unrelated

205 families (F1 \& F14).

206 In addition, a novel variant in $O T O G$ not included in the GBA was found in two cases

207 from a 15th family (F34). This variant, located in exon 18 (chr11:17594747C>A), was

208 found in heterozygous state affecting the sequence of the $\mathrm{C} 8$ domain. The distribution

209 of the variants found in $O T O G$ is scattered across the gene sequence (Figure 2).

\section{Hearing profile in familial patients with rare variants in $O T O G$}

211 The hearing profile for the 14 patients (3 males, 11 females) with rare variants in OTOG

212 gene was studied (see Figure 2, Supplemental Digital Content 2, which shows the pure

213 tone audiograms for these patients). Ten of them showed bilateral hearing loss, 3 had

214 left-sided hearing loss and only 1 patient shown right-sided SNHL (see Table 1,

215 Supplemental Digital Content 1, which summarizes the clinical information of the

216 familial MD cases carrying variants in $O T O G$ gene). From these 14 patients, 16 ears

217 from 12 patients showed a flat shaped audiogram (57.1\%), 5 ears from 5 patients

218 showed a ski-slope shaped audiogram (17.8\%), 3 ears from 3 patients showed a reverse-

219 slope shaped (10.7\%) and 4 ears had a normal pure-tone audiogram (14.2\%). 
220 A regression analysis was done to estimate the hearing loss at onset and the outcome for

221 each frequency. We found a negative correlation at $1000 \mathrm{~Hz}\left(\mathrm{R}^{2}=.143 ; p=.033\right)$ and

$2222000 \mathrm{~Hz}\left(\mathrm{R}^{2}=.246 ; p=.004\right)$. There was no statistical correlation at $125 \mathrm{~Hz}, 250 \mathrm{~Hz}, 500$

$223 \mathrm{~Hz}, 4000 \mathrm{~Hz}$ nor $8000 \mathrm{~Hz}$, suggesting no progression at these frequencies (Figure 3).

224 The age of onset of the symptoms was $41.93 \pm 8.66$ and the estimated hearing loss at

225 onset was $62.14 \pm 12.83$ for low frequencies $(125-250-500 \mathrm{~Hz})$ and $58.75 \pm 14.1$ for high

226 frequencies $(1000-2000-4000 \mathrm{~Hz})$.

\section{Early onset sporadic MD}

228 The same analytical pipeline was used in a series of patients with sporadic MD with an 229 age of onset younger than 35 (see Figure 3, Supplemental Digital Content 2). For the

230 SRVA, we found 60 nonsynonymous or splice site SNV with MAF $<0.001$ and CADD

$231>15$ in SNHL genes. Among them, one variant was found in two sporadic cases and 232 another variant was also found in a familial case. The rest of the SNV were considered 233 simplex variants found in singletons and none of them were homozygous (see Table 6, 234 Supplemental Digital Content 1, which shows the rare variants found in the SRVA for 235 SMD cases).

236 A heterozygous nonsynonymous SNV in $O T O G$ gene was found in two unrelated 237 sporadic MD cases (S1 and S23). The variant chr11:17632279C > T (rs779658224;

238 p.A1823V) is located in exon 35 of the canonical transcript of $O T O G$ gene and it is a

239 variant of uncertain significance (VUS) according to the ACMG and AMP guidelines.

240 This variant has a MAF=.0005 in the NFE population from ExAC and it is not

241 described in the Spanish population from the CSVS. In addition, a heterozygous

242 nonsynonymous SNV in OTOGL gene was found in one sporadic case and in one

243 familial case (S27 and F31). The variant chr12:80752642T > G (rs145929269; 
244 p.C2068G) is located in exon 51 of the canonical transcript of OTOGL gene

245 (ENST00000458043). This region encodes a cysteine-rich region and this variant was

246 also classified as a VUS according to the ACMG and AMP guidelines.

247 For the GBA, we found 12 rare SNV in OTOG gene in patients with early onset MD

248 (see Table 7, Supplemental Digital Content 1). However, in contrast with the results

249 obtained in FMD cases, there was not an excess of rare variants in this gene against

250 neither the NFE population from ExAC (OR=2.1(1.2-3.7), $p=.11)$ nor Spanish

251 population $(\mathrm{OR}=2(1.1-3.5), p=.20)$ (Figure 2). 


\section{DISCUSSION}

253 Familial MD has an AD inheritance with incomplete penetrance (Morrison et al. 2009;

254 Requena et al. 2014), and few genes have been involved in singular families (Requena

255 et al. 2015; Martín-Sierra et al. 2016; Martín-Sierra et al. 2017). In this study, we have

256 found an enrichment of rare missense variants in several unrelated patients with FMD in

257 the OTOG gene. These variants were observed in 15 of 46 non-related families (33\%

258 familial cases). Seven of the 15 families with rare variants in $O T O G$ showed incomplete

259 penetrance (47\%) and partial syndromes (episodic vertigo or hearing loss) were found in

260 relatives from 5 of 15 families (Morrison et al. 2009; Requena et al. 2015; Martín-Sierra

261 et al. 2016; Martín-Sierra et al. 2017). Most of these rare variants were found in 2, 3 or

2624 unrelated individuals from different families with MD and they were considered

263 multiplex variants. However, the majority of the variants in OTOG found in non-

264 familial patients with early onset were not observed in other sporadic cases (singletons

265 variants).

266 OTOG, which encodes otogelin, was described for first time by Cohen-Salmon et al

267 (Cohen-Salmon et al. 1997). Otogelin is a 2925 amino acid protein

268 (ENST00000399391) constituted by several vWD and C8 domains, and a cysteine knot-

269 like domain in its C-terminal. It is mainly expressed in acellular structures which cover

270 the sensory inner ear epithelia: the tectorial membrane, the otoconial membranes and

271 the cupula over the cristae ampullaris of the semicircular canals. Because of its

272 localization in the extracellular structures overlying the stereocilia of the hair cells

273 involved in the mechanotransduction of sound and acceleration, this structural protein

274 plays an important role in both auditory and vestibular functions (Schrauwen et al.

275 2016). 
276 The effects of variants in otogelin were first demonstrated in the orthologous gene in a

277 mouse model. Three mouse models have been generated for evaluating the phenotypic

278 changes resulting from $O T O G$ variants. In the Otog ${ }^{\mathrm{tm} 1 \mathrm{Prs}}$ model, authors inactivated

279 Otog by deleting the first three exons. Vestibular dysfunction was detected at P4 in

$280 \mathrm{Otog}^{-/-}$, observing anomalies in the saccule and utricule. The auditory function was

281 evaluated by Pleyer reflex, showing profound hearing impairment. The Otog ${ }^{+-}$mice did

282 not present any anomalies (Simmler et al. 2000a). The second model is the twister (twt)

283 mice, mice with a spontaneous recessive mutation entailing absence of Otog expression.

284 Similarly to Otog ${ }^{\text {tm1Prs }}$, in Otog ${ }^{\text {twt }}$ the vestibular dysfunction was detected at P4, and the

285 hearing loss was progressive and moderate to severe/profound (Simmler et al. 2000b).

286 The last mouse model published is the otogelin ENU-induced mouse model. In this

287 model, a homozygous variant at the splice donor site of intron $29, \mathrm{Otog}^{\mathrm{vbd} / \mathrm{vbd}}$, cause a

288 frame-shift and a premature codon. Otog ${ }^{\mathrm{vbd} / \mathrm{vbd}}$ mice shown abnormal hearing and

289 vestibular functions (El Hakam Kamareddin et al. 2015).

290 Four variants have been described in OTOG gene causing DFNB18B. Schraders et al.

291 were the first to describe causative variants in OTOG. A homozygous 1bp deletion,

292 c.5508delC (p.Ala1838Profs*31) in four related patients, and two compound-

293 heterozygous variants, c.6347C>T (p.Pro2116Leu) and c.6559C>T (p.Arg2187*) in

294 other two related patients, were described to cause hearing loss and vestibular

295 dysfunction (Schraders et al. 2012). More recently, a homozygous nonsense variant

296 c.330C > G (p.Tyr110*) in a Korean patient has been identified, showing early-onset

297 mild hearing loss without vestibular dysfunction (Yu et al. 2019).

298 In contrast to studies mentioned above, $O T O G$ variants found in this study were all in

299 heterozygous state and, despite 6 FMD cases and 3 SMD cases studied had two or more

300 variants, compound heterozygous variants could not be demonstrated because samples 
301 from the parents were not available and $O T O G$ variant segregation could not be fully

302 assessed in each family. However, the variants chr11:17574758G $>$ A and

303 chr11:17663747G $>$ A found in F14 were also identified in his mother, the variants

304 chr11:17578774G > A and chr11:17632921C > T found in F5 were also identified in her

305 sister (II-7), and a novel variant chr11:17594747C>A not considered for the GBA were

306 found in F34 and her brother. Furthermore, variants located in untranslated regions

307 (UTRs) and promoter regions, which modulate gene expression and different protein

308 features (Chatterjee \& Pal 2009; Buckland 2006), could not be evaluated because of the

309 study design. Altogether, the results obtained by GBA suggested a different genetic

310 architecture in FMD cases and SMD cases, since the enrichment of rare variants in

311 OTOG gene was only found in FMD cases and most of the variants found in sporadic

312 cases with early onset were singletons (not observed in multiple individuals).

313 Each region of the cochlea is specifically stimulated by a specific frequency. Thus, the

314 base of the cochlea mainly responds to high-frequency sounds, whereas the apex

315 responds to low-frequency sounds, frequencies mostly affected in MD (Robles \&

316 Ruggero 2017; Nakashima et al. 2016). Of note, otogelin shows a tonotopic gene

317 expression in mice (Yoshimura et al. 2014). OTOG gene showed a 2.43-fold change in

318 expression for apex vs base, making this gene a possible candidate for SNHL in MD. In

319 addition, an RNA-seq study of the inner ear from patients with normal hearing showed a

320 high expression of OTOG gene in the vestibule (Schrauwen et al. 2016), which could

321 explain the vestibular dysfunction in patients with pathogenic variants in this gene.

322 The audiograms of FMD patients who carried rare variants in $O T O G$ gene showed a

323 moderate-to-severe flat hearing loss $\approx 60 \mathrm{~dB}$ since the first years of onset involving all

324 frequencies. Low-frequency hearing had slight variations throughout the years, while a

325 negative correlation was found at $\operatorname{mid}(1000 \mathrm{~Hz})$ and high-frequency $(2000 \mathrm{~Hz})$ hearing. 
326 Data from F14 were considered as an outlier and discarded because his hearing profile

327 was not comparable to the rest of FMD patients (see Figure 2, Supplemental Digital

328 Content 2). Since all frequencies are involved since the onset of the disease, we can

329 speculate that the damage of the tectorial membrane mediated by mutations in otogelin

330 will involve the entire cochlea from base to apex.

331 According to our results, the clinical picture of patients with mutations in $O T O G$ would

332 be a female of 43 years old with sudden or rapidly progressive flat SNHL around $60 \mathrm{~dB}$

333 and vertigo attacks with a family history of MD, vertigo or early onset SNHL.

334 In conclusion, we have found an enrichment of rare missense variants in the OTOG

335 gene in FMD cases. These findings support a multiallelic contribution in MD, where

336 OTOG gene seems to be playing a relevant role in the pathophysiology of hearing and

337 vestibular functions in MD. 


\section{Acknowledgements}

339 We thank to all participants of the Meniere's Disease Consortium for recruiting patients

340 with familial MD and their relatives. Pablo Roman-Naranjo is a PhD student in the

341 Biomedicine Program at Universidad de Granada and his salary was supported by

342 ASMES (Asociación Sindrome de Meniere España). JALE conceived the study design

343 and recruited all clinicians involved in the Meniere's Disease Consortium to

344 characterize families with MD at different sites (AS-V, IA, MCM, JME-S, JCA-D, AB-

345 C, PP-V). PR-N and AG-M conducted DNA extractions, WES and all bioinformatics

346 analyses. PR-N and JALE drafted the manuscript and all authors approved the final

347 version of the manuscript.

348 Jose Antonio Lopez Escamez (JALE) is partially funded by INT18/00031 from ISCIII.

349 This study was funded by the Luxembourg National Research Fund

350 INTER/Mobility/17/11772209 Grant and EF-0247-2017 from Andalusian Health

351 Government to JALE.

352 Authors declare no conflict of interest. 


\section{References}

354 Adams, D.R., Eng, C.M. (2018). Next-Generation Sequencing to Diagnose Suspected

$355 \quad$ Genetic Disorders. N. Engl. J. Med., 379, 1353-1362.

Azaiez, H., Booth, K.T., Ephraim, S.S., et al. (2018). Genomic Landscape and Mutational Signatures of Deafness-Associated Genes. Am. J. Hum. Genet., 103,

Belinchon, A., Perez- Garrigues, H., Tenias, J.M., et al. (2011). Hearing assessment in Menière's disease. Laryngoscope, 121, 622-626.

Birgerson, L., Gustavson, K.H., Stahle, J. (1987). Familial Menière’s disease: a genetic investigation. Am. J. Otol., 8, 323-6.

Buckland, P.R. (2006). The importance and identification of regulatory polymorphisms and their mechanisms of action. Biochim. Biophys. Acta - Mol. Basis Dis.

Van Camp G., S.R.J.H. (2018). Hereditary Hearing Loss Homepage. Available at: https://hereditaryhearingloss.org.

Chatterjee, S., Pal, J.K. (2009). Role of 5'- and 3'-untranslated regions of mRNAs in

Cha, Y.-H., Kane, M.J., Baloh, R.W. (2008). Familial Clustering of Migraine, Episodic Vertigo, and Ménière's Disease. Otol. Neurotol., 29, 93-96.

Cohen-Salmon, M., El-Amraoui, A., Leibovici, M., et al. (1997). Otogelin: a Sci. U. S. A., 94, 14450-5.

Cooper, D.N., Krawczak, M., Polychronakos, C., et al. (2013). Where genotype is not predictive of phenotype: towards an understanding of the molecular basis of 
reduced penetrance in human inherited disease. Hum. Genet., 132, 1077-1130.

377

Dopazo, J., Amadoz, A., Bleda, M., et al. (2016). 267 Spanish Exomes Reveal Population-Specific Differences in Disease-Related Genetic Variation. Mol. Biol. Evol., 33, 1205-1218.

Espinosa-Sanchez, J.M., Lopez-Escamez, J.A. (2016). Menière's disease. In Handbook of clinical neurology. (pp. 257-277).

Frejo, L., Martin-Sanz, E., Teggi, R., et al. (2017). Extended phenotype and clinical subgroups in unilateral Meniere disease: A cross-sectional study with cluster analysis. Clin. Otolaryngol., 42, 1172-1180.

Frejo, L., Soto-Varela, A., Santos-Perez, S., et al. (2016). Clinical Subgroups in Bilateral Meniere Disease. Front. Neurol., 7, 182.

Gallego-Martinez, A., Requena, T., Roman-Naranjo, P., et al. (2019). Excess of Rare Missense Variants in Hearing Loss Genes in Sporadic Meniere Disease. Front. Genet., 10.

El Hakam Kamareddin, C., Magnol, L., Blanquet, V. (2015). A new Otogelin ENU mouse model for autosomal-recessive nonsyndromic moderate hearing impairment. Springerplus, 4, 730.

Klar, J., Frykholm, C., Friberg, U., et al. (2006). A Meniere's disease gene linked to chromosome 12p12.3. Am. J. Med. Genet. Part B Neuropsychiatr. Genet., 141B, $463-467$.

Kousi, M., Katsanis, N. (2015). Genetic Modifiers and Oligogenic Inheritance. Cold Spring Harb. Perspect. Med., 5, a017145-a017145.

Lee, J.-M., Wheeler, V.C., Chao, M.J., et al. (2015a). Identification of Genetic Factors 
that Modify Clinical Onset of Huntington's Disease. Cell, 162, 516-526.

400

401

402

Lee, J.M., Kim, M.J., Jung, J., et al. (2015b). Genetic aspects and clinical characteristics of familial meniere's disease in a South Korean population. Laryngoscope, 125, $2175-2180$.

Lek, M., Karczewski, K.J., Minikel, E. V., et al. (2016). Analysis of protein-coding genetic variation in 60,706 humans. Nature, 536, 285-291.

Lopez-Escamez, J.A., Carey, J., Chung, W.-H., et al. (2015). Diagnostic criteria for Menière's disease. J. Vestib. Res., 25, 1-7.

Lubbe, S.J., Escott-Price, V., Gibbs, J.R., et al. (2016). Additional rare variant analysis in Parkinson's disease cases with and without known pathogenic mutations: evidence for oligogenic inheritance. Hum. Mol. Genet., 25:5483-9.

Martín-Sierra, C., Gallego-Martinez, A., Requena, T., et al. (2017). Variable expressivity and genetic heterogeneity involving DPT and SEMA3D genes in autosomal dominant familial Meniere's disease. Eur. J. Hum. Genet., 25, 200-207.

Martín-Sierra, C., Requena, T., Frejo, L., et al. (2016). A novel missense variant in $P R K C B$ segregates low-frequency hearing loss in an autosomal dominant family with Meniere's disease. Hum. Mol. Genet., 25, 3407-3415.

Morrison, A.W., Bailey, M.E.S., Morrison, G.A.J. (2009). Familial Ménière’s disease: clinical and genetic aspects. J. Laryngol. Otol., 123, 29-37.

Nakashima, T., Pyykkö, I., Arroll, M.A., et al. (2016). Meniere's disease. Nat. Rev. Dis. Prim., 2, 16028.

Ohmen, J.D., White, C.H., Li, X., et al. (2013). Genetic Evidence for an Ethnic Diversity in the Susceptibility to Ménière's Disease. Otol. Neurotol., 34, 1336- 
1341.

423 Requena, T., Cabrera, S., Martin-Sierra, C., et al. (2015). Identification of two novel mutations in FAM136A and DTNA genes in autosomal-dominant familial Meniere's disease. Hum. Mol. Genet., 24, 1119-1126.

Requena, T., Espinosa $\square$ Sanchez, J.M., Cabrera, S., et al. (2014). Familial clustering and genetic heterogeneity in Meniere's disease. Clin. Genet., 85, 245-252.

Richards, S., Aziz, N., Bale, S., et al. (2015). Standards and guidelines for the interpretation of sequence variants: a joint consensus recommendation of the American College of Medical Genetics and Genomics and the Association for

Robles, L., Ruggero, M.A. (2017). Mechanics of the Mammalian Cochlea. Physiol. Rev. vestibular syndromes. Curr. Opin. Neurol., 31, 1.

Rouillard, A.D., Gundersen, G.W., Fernandez, N.F., et al. (2016). The harmonizome: a collection of processed datasets gathered to serve and mine knowledge about genes and proteins. Database, 2016, baw100. Res., 333, 266-274. 
differences in minor allele frequency to recategorize reported pathogenic deafness variants. Am. J. Hum. Genet., 95, 445-53.

Simmler, M.-C., Cohen-Salmon, M., El-Amraoui, A., et al. (2000a). Targeted disruption of Otog results in deafness and severe imbalance. Nat. Genet., 24, 139-143.

Simmler, M.C., Zwaenepoel, I., Verpy, E., et al. (2000b). Twister mutant mice are defective for otogelin, a component specific to inner ear acellular membranes.

Suwinski, P., Ong, C., Ling, M.H.T., et al. (2019). Advancing Personalized Medicine Through the Application of Whole Exome Sequencing and Big Data Analytics. Front. Genet., 10, 49.

Szczepek, A.J., Frejo, L., Vona, B., et al. (2019). Recommendations on Collecting and Storing Samples for Genetic Studies in Hearing and Tinnitus Research. Ear Hear.,

Williams, H.J., Hurst, J.R., Ocaka, L., et al. (2016). The use of whole-exome 40, 219-226.

Tyrrell, J.S., Whinney, D.J.D., Ukoumunne, O.C., et al. (2014). Prevalence, Associated Factors, and Comorbid Conditions for Ménière's Disease. Ear Hear., 35, e162sequencing to disentangle complex phenotypes. Eur. J. Hum. Genet., 24, 298-301.

Yoshimura, H., Takumi, Y., Nishio, S.Y., et al. (2014). Deafness gene expression patterns in the mouse cochlea found by microarray analysis. PLoS One. causes prelingual mild hearing loss without vestibular dysfunction. Eur. J. Med. Genet., 62, 81-84. 
bioRxiv preprint doi: https://doi.org/10.1101/771527; this version posted September 21, 2019. The copyright holder for this preprint (which was not certified by peer review) is the author/funder, who has granted bioRxiv a license to display the preprint in perpetuity. It is made available under aCC-BY-NC-ND 4.0 International license.

468 Zheng, Q.Y., Yan, D., Ouyang, X.M., et al. (2005). Digenic inheritance of deafness

469 caused by mutations in genes encoding cadherin 23 and protocadherin 15 in mice

$470 \quad$ and humans. Hum. Mol. Genet., 14, 103-111. 


\section{Figure legends}

Figure 1: Flowchart summarizing the bioinformatic analysis on familial MD cases. On the left, single rare variant analysis (SRVA) and prioritization pipeline. On the right, the gene burden analysis (GBA) pipeline. SNV, single nucleotide variants; CADD, Combined Annotation Dependent Depletion Score.

Figure 2: Variants distribution across OTOG gene domains. On the upper part, variants which were found in familial Meniere disease (FMD) cases. On the bottom part, variants which were found in sporadic Meniere disease (SMD) cases. Yellow-colored variants indicate variants found in only one case, whereas red-colored variants represent variants found in 2 or more cases in a cohort. vWD, von Willebrand factor type D domain; T, Trypsin inhibitor-like domain; Abf, Alpha-L-arabinofuranosidase B domain; CT, Cysteine knot domain.

Figure 3: Scattered plot showing air conduction hearing thresholds obtained and the duration of the disease for each frequency in familial MD cases. Regression equations and estimated hearing loss at the onset are displayed below the charts.

\section{Supplemental Digital Content}

Supplemental Digital Content 1.docx

Supplemental Digital Content 2.docx 
Table 1. Rare Variants Found in the Gene Burden Analysis in OTOG Gene for Familial MD Cases.

\begin{tabular}{|c|c|c|c|c|c|c|c|c|c|}
\hline Variant position & Exon & Families & Sporadic cases & $\begin{array}{l}\text { MAF } \\
\text { FMD }\end{array}$ & $\begin{array}{c}\text { MAF } \\
\text { ALL MD }\end{array}$ & $\begin{array}{l}\text { MAF } \\
\text { NFE }\end{array}$ & $\begin{array}{l}\text { MAF } \\
\text { CSVS }\end{array}$ & CADD & Domain \\
\hline $11: 17574758 \mathrm{G}>\mathrm{A}$ & 4 & F1; F14 & - & $0.041(3 / 73)$ & $0.028(3 / 109)$ & 0.00080 & 0.0033 & 24.8 & vWD \\
\hline $11: 17578774 \mathrm{G}>\mathrm{A}$ & 7 & F2; F3; F4; F5 & S24 & $0.068(5 / 73)$ & $0.055(6 / 109)$ & 0.0090 & 0.017 & 15.95 & vWD \\
\hline $11: 17621218 \mathrm{C}>\mathrm{T}$ & 30 & F6; F7 & - & $0.027(2 / 73)$ & $0.018(2 / 109)$ & 0.0026 & 0.0033 & 34 & $\mathrm{C} 8$ \\
\hline $11: 17627548 \mathrm{G}>\mathrm{A}$ & 32 & F14 & - & $0.012(1 / 73)$ & $0.009(1 / 109)$ & 0.0056 & 0.0054 & 23.6 & Abf \\
\hline $11: 17656672 \mathrm{G}>\mathrm{A}$ & 45 & F10 & S9 & $0.013(1 / 73)$ & $0.018(2 / 109)$ & 0.0034 & 0.0039 & 31 & - \\
\hline $11: 17663747 \mathrm{G}>\mathrm{A}$ & 52 & F1; F13; F14 & S7 & $0.055(4 / 73)$ & $0.046(5 / 109)$ & 0.0058 & 0.0054 & 19.41 & - \\
\hline $11: 17667139 \mathrm{G}>\mathrm{C}$ & 54 & F9; F11; F12 & S12; S20 & $0.082(6 / 73)$ & $0.073(8 / 109)$ & 0.019 & 0.017 & 27.2 & $\mathrm{CT}$ \\
\hline
\end{tabular}

* This novel variant was not included in the gene burden analysis. Abbreviations: MAF FMD, minor allele frequency in familial MD; MAF ALL MD, minor allele frequency in all familial and non-familial MD cases ; NFE, Minor allele frequency in non-Finnish European population; CSVS, Collaborative Spanish Variant Server; Abf, alpha-L-arabinofuranosidase B domain; CADD, Combined Annotation Dependent Depletion Score; CT: cysteine knot domain; vWD, von Willebrand factor type D domain. 


\section{SNV in SNHL genes}

\section{Quality control}

SRVA analysis

\section{SNV}

\section{GBA analysis}

Nonsynonymous/splice variants with $\mathrm{MAF}<0.001$.

\section{SNV}

\section{SNV}

CADD value $>15$

75 SNV

Described in public

L._._. databases 


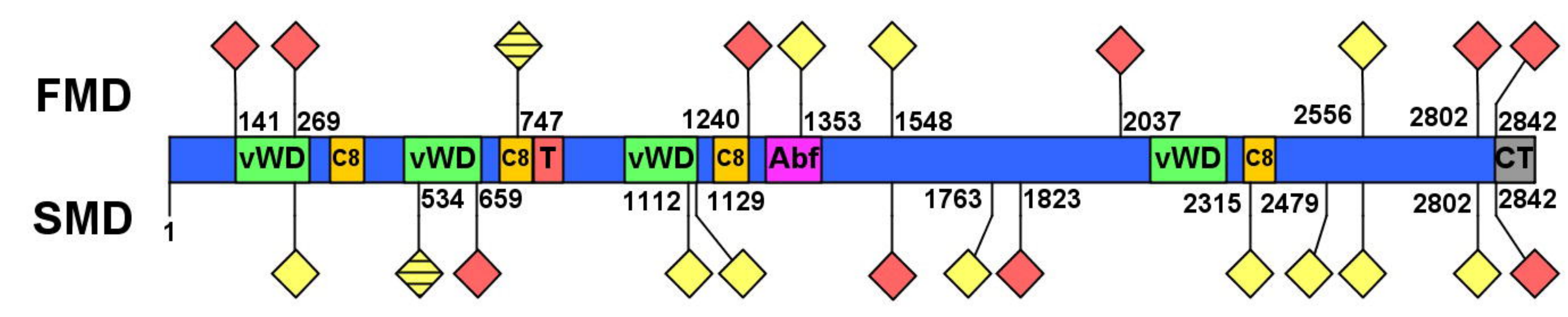



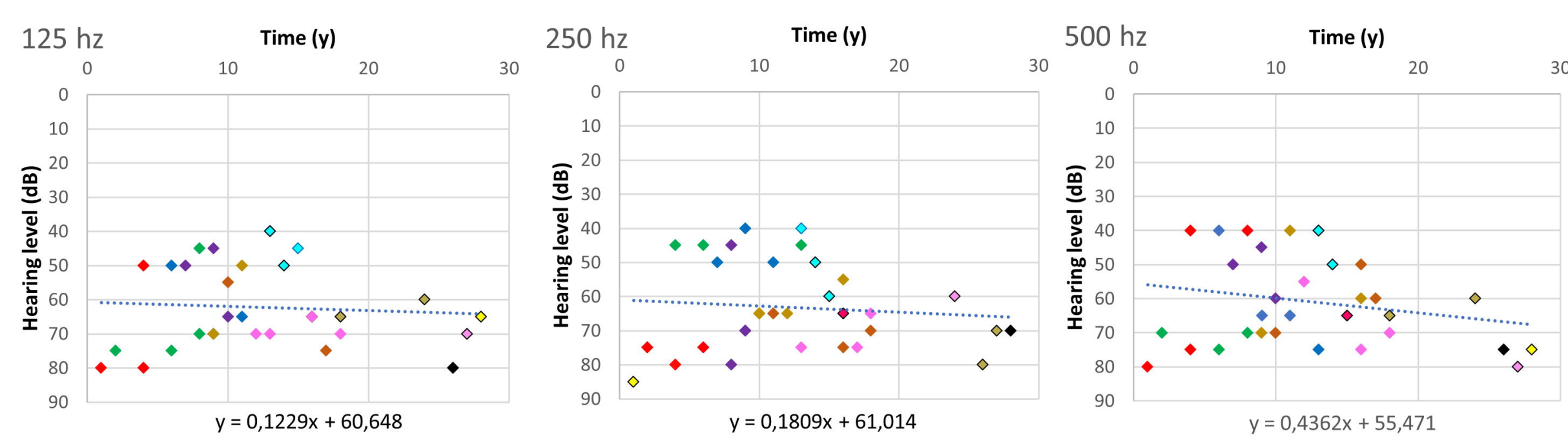

$1000 \mathrm{hz}$
0

Time (y)
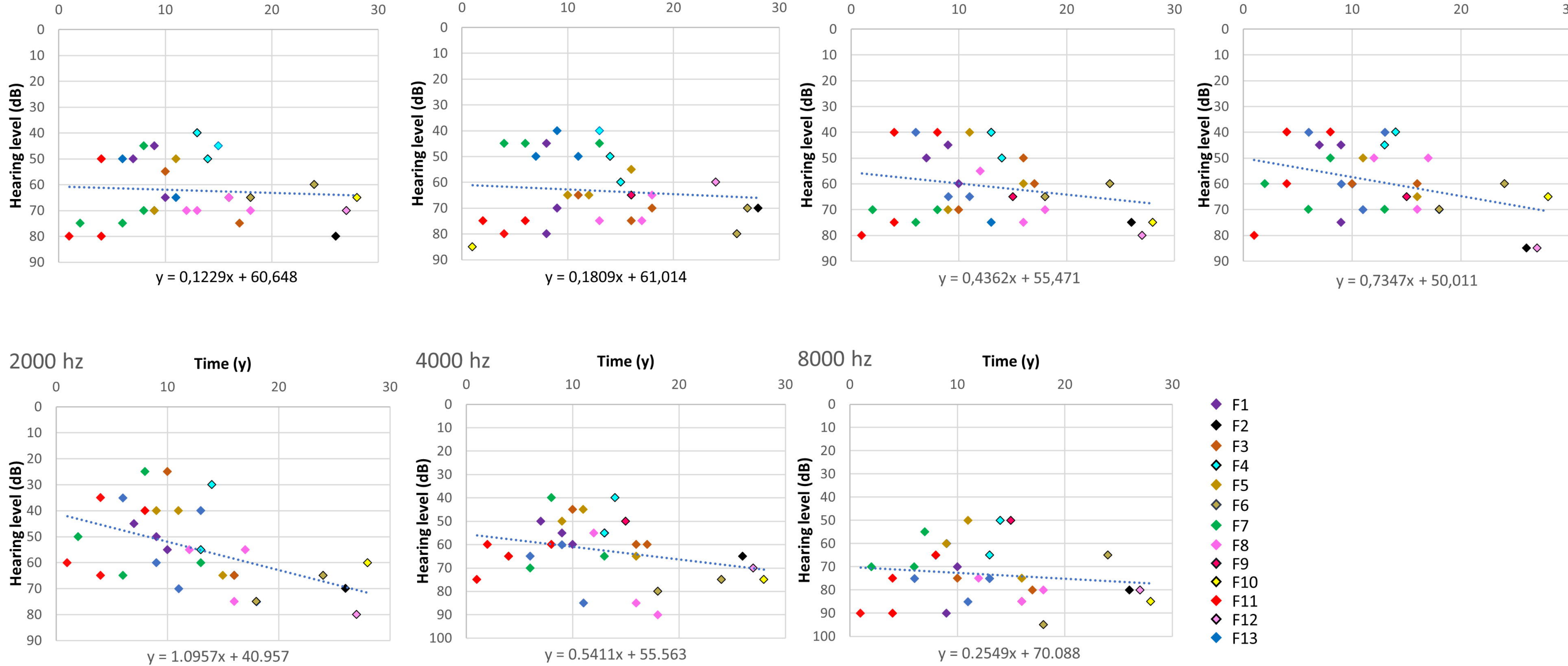

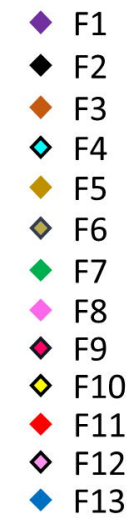

. 